\title{
REFERENCE
}

\section{Molecular Dynamics Study of the Solubility of Oxygen in Liquid Pyridine}

Mountain, R. D.

U. S. DEPARTMENT OF COMMERCE Technology Administration

National Institute of Standards and Technology

Gaithersburg, MD 20899

\section{QC}

100

.456

\# 7075

2003

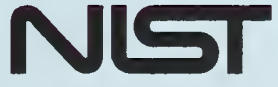

National Institute of Standards and Technology

Technology Administration

U.S. Department of Commerce 



\section{Molecular Dynamics Study of the Solubility of Oxygen in Liquid Pyridine}

Mountain, R. D.

U. S. DEPARTMENT OF COMMERCE Technology Administration National Institute of Standards and Technology

Gaithersburg, MD 20899

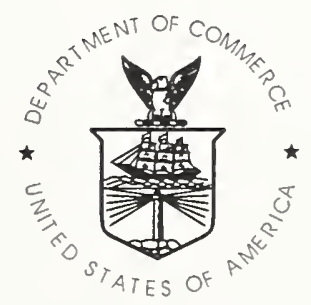

U.S. DEPARTMENT OF COMMERCE Donald L. Evans, Secretary TECHNOLOGY ADMINISTRATION Phillip J. Bond, Under Secretary for Technology NATIONAL INSTITUTE OF STANDARDS AND TECHNOLOGY

Arden L. Bement, Jr., Director 



\title{
Molecular dynamics study of the solubility of oxygen in liquid pyridine
}

\author{
Raymond D. Mountain $†$ \\ Physical and Chemical Properties Division \\ National Institute of Standards and Technology \\ 100 Bureau Drive Stop 8380 \\ Gaithersburg, MD 20899-8380
}

\begin{abstract}
The solubility of oxygen in liquid pyridine is estimated using molecular dynamics to evaluate the chemical potential of the gas molecule in the liquid. The Kirkwood charging method is used with empirical potentials that generate reliable equations of state for the pure components and sixth power combining rules to obtain the oxygen-pyridine interactions. The computed value of the solubility is 120 times smaller than the experimental value when combining rule parameters are used. A set of potential parameters that result in the predicted solubility close to the experimental value are not accessible via the usual combining rules.
\end{abstract}

Key words: Kirkwood charging, combining rules, molecular dynamics, oxygen, pyridine, solubility.

\section{Introduction}

Molecular dynamics and Monte Carlo simulation methods make it possible to calculate fluid properties provided suitable potential functions are available. This method is particularly attractive when the costs of experimental work are large due to extreme/hazardous conditions or when samples are very expensive and when the interest is to screen for particular properties among several systems. The solubility of oxygen in organic liquids where the risk of fire is a significant hazard, is an example where simulation provides an attractive alternative to laboratory work. In this paper, we discuss the use of molecular dynamics simulation to determine the solubility of oxygen in liquid pyridine. This is the first step in developing a protocol for making solubility calculations of gases in polar liquids routine. We employ the Kirkwood charging method [1] as discussed in the next section. As in any simulation, the choice of potential functions is important. The potentials used here are described in the next section along with the simulation details. The results are presented

† email: raymond.mountain@nist.gov 
in section 3 and some discussion on how these results might be improved is found in the final section.

\section{Models and methods}

The solubility, $s$, of a gas in a liquid is the amount of gas dissolved to saturation relative to the total number of molecules in the liquid. [2] It is equal to the mole fraction of the gas, $x_{g}$, in the liquid. The condition to be satisfied is that the chemical potential of the gas molecule dissolved in the liquid equals the chemical potential of the gas molecule in the vapor and therefore depends on the pressure in the gas phase. The chemical potential in the vapor phase, $\mu_{g}$ can be determined from the equation of state of the gas and the ideal gas chemical potential. [3] The chemical potential of the gas molecule in the liquid can be obtained by evaluating a "charging potential" integral as discussed by Swope and Andersen [4] and more recently in a different context by Ferrario, et al. [5]

Suppose the interaction between the gas molecule and the liquid molecules is described by a potential function $U(\lambda)$ where $\lambda$ is a charging parameter that can vary from 0 to 1 . Typically, $U(0)=0$ and $U(\lambda)$ increases to the full solvent-solute interaction for $\lambda=1$. It follows that the chemical potential of the gas molecule in the liquid, $\mu_{l}$ is

$$
\mu_{l}=k_{B} T \ln \left(n_{s} \Lambda^{3}\right)+\int_{0}^{1}\langle\partial U(\lambda) / \partial \lambda\rangle_{\lambda} d \lambda
$$

where $n_{s}$ is the number density of the solute in the solution, $\Lambda$ is the thermal deBroglie wavelength, and $\langle\ldots\rangle_{\lambda}$ indicates an ensemble average in the NPT ensemble of the enclosed quantity with the charging parameter set to $\lambda$. If the gas in the vapor phase is sufficiently dilute to be considered an ideal gas with number density $n_{v}$, equating the chemical potentials of the solute in the two phases yields

$$
\ln \left(n_{s} / n_{v}\right)=-\frac{1}{k_{B} T} \int_{0}^{1}\langle\partial U(\lambda) / \partial \lambda\rangle_{\lambda} d \lambda
$$

with $k_{B} T$ the product of Boltzmann's constant times the temperature. The solubility is therefore

$$
s=x_{g}=\frac{n_{s}}{n_{s}+n_{l}}
$$

where $n_{l}$ is the number density of the liquid. The explicit $\lambda$ variation of the solvent-solute interaction used here has the overall form of

$$
U(\lambda, r)=\lambda^{2} \phi\left(\alpha\left(1-\lambda^{2}\right)+r\right)
$$

where factor $\alpha\left(1-\lambda^{2}\right)$ prevents singular behavior for the small $r$ separations that occur for small values of $\lambda$. [6] The explicit form for the oxygen-pyridine interaction potential is 
described below. The modifications to eq. 2 needed when the gas is too dense to be treated as an ideal gas are discussed in the appendix. Any complications due to the presence of solute molecules in the gas phase are ignored.

It is important to realize that the path in the $\lambda$-parameter space followed in eq. 1 is arbitrary provided that $U(0)=0$ and $U(1)$ is the full solute-solvent interaction. This is because differences in thermodynamic potentials depend only on the states and not on the equilibrium path followed between the states.

The charging parameter approach avoids one of the potential problems of small system size when the solubility is expected to be small. In the Widom insertion method [7] and in the NPT ensemble variant of that method [8] the acceptance rate of insertions per attempted insertion would be on the order of 0.0005 if the experimental value for the solubility of oxygen in pyridine [9] were realized. This would make it difficult to obtain statistically significant results without resorting to extremely long simulations. The same concerns apply to Gibbs ensemble methods since the number of oxygen molecules present in the pyridine liquid would be small compared with the total number of molecules in the liquid. [10]

The simulations are performed using NPT molecular dynamics [11] for a system of 215 rigid pyridine molecules and 1 rigid oxygen molecule. The dynamical degrees of freedom of the molecules are evolved using a variant of the velocity Verlet algorithm with quaternions used to describe the orientation of the molecules. [12] The equations of motion were integrated using a time step of $1 \mathrm{fs}$. The temperature of the system is held at $303 \mathrm{~K}$ and the pressure is set to $0.1 \mathrm{MPa}$. The Ewald summation method is used to account for the long range part of the Coulomb ineractions and a correction is made for the long range part of the $\mathrm{r}^{-6}$ interaction terms as well. [13] More detail about simulation procedures, including uncertainty estimation methods and a convergence test, are found in the appendix.

The pyridine model treats the molecule as a rigid, all-atom object with the geometry of the isolated molecule [14] and with site-site interactions of the form

$$
\phi_{a b}(r)=\epsilon(a b)\left[2\left(r_{0}(a b) / r\right)^{9}-3\left(r_{0}(a b) / r\right)^{6}\right]+q_{a} q_{b} / r
$$

where $\epsilon(a b)$ and $r_{0}(a b)$ are appropriate for sites $a$ and $b$ on distinct molecules and the sites are separated by a distance $r$. The sites on the pyridine molecule are indicated in Fig. 1. The charges and the $\epsilon$ and $r_{0}$ values were initially taken from published sources [15] for the charges and for the other parameters [16]. Sixth power combining rules [17] were used to obtain the unlike site interaction parameters, namely

$$
r_{0}(a b)=\left[\left(r_{0}(a a)^{6}+r_{0}(b b)^{6}\right) / 2\right]^{1 / 6}
$$


and

$$
\epsilon(a b)=2 \sqrt{\epsilon(a a) \epsilon(b b)}\left[\frac{\left(r_{0}(a a) r_{0}(b b)\right)^{3}}{r_{0}(a a)^{6}+r_{0}(b b)^{6}}\right] .
$$

These initial values of the interaction parameters were then adjusted so that the correct value for the density of liquid pyridine at $303 \mathrm{~K}$ and $0.1 \mathrm{MPa}\left(972 \mathrm{~kg} \cdot \mathrm{m}^{-3}\right)$ was obtained within $0.1 \%$. [18] For the $10 \mathrm{MPa}$ state, these parameters overestimate the density by $1.4 \%$. The values of the 9-6 potential parameters are listed in Table 1.

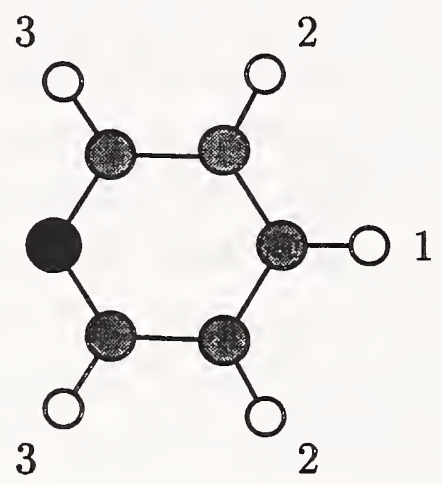

Fig. 1. The pyridine molecule with equivalent sites are shown here. The nitrogen site is the black filled circle, the carbon sites are the gray filled circles and the hydrogen sites are the open circles. The labels 1, 2, and 3 identify the three types of carbon and hydrogen sites with distinct charges as indicated in Table 1.

Table 1. The potential parameters for pyridine and pyridine-oxygen interactions. The oxygen-oxygen parameters are included for completeness.

\begin{tabular}{|c|c|c|c|r|}
\hline a-b pair & $\epsilon(\mathrm{ab}) / \mathrm{k}_{B}, \mathrm{~K}$ & $\mathrm{r}_{0}(\mathrm{ab}), \mathrm{nm}$ & Site number & \multicolumn{1}{c|}{$\mathrm{q}$} \\
\hline C-C & 21.77 & 0.4466 & $\mathrm{C} 1$ & 0.25361 \\
H-H & 6.94 & 0.3299 & $\mathrm{C} 2$ & -0.50028 \\
N-N & 53.92 & 0.4161 & $\mathrm{C} 3$ & 0.47827 \\
C-N & 33.51 & 0.4327 & $\mathrm{~N}$ & -0.65797 \\
C-H & 8.53 & 0.4080 & $\mathrm{H} 1$ & 0.06774 \\
H-N & 15.45 & 0.3847 & $\mathrm{H} 2$ & 0.17813 \\
O-C & 21.48 & 0.4143 & $\mathrm{H} 3$ & 0.01219 \\
O-H & 14.24 & 0.3931 & & \\
O-N & 37.51 & 0.3467 & & \\
O-O & 31.30 & 0.3602 & & \\
\hline
\end{tabular}


The oxygen molecule is also treated as a rigid object with the interatomic separation of $0.1208 \mathrm{~nm}$. [19] To be consistent with the pyridine interactions, the oxygen-oxygen interaction is also of the form of a 9-6 interaction with an additional quadrupole-quadrupole interaction. Interaction sites are located at the oxygen sites and at the point quadrupole site located at the midpoint between the oxygen sites and is assigned the experimental value of $-0.4 \times 10^{-26}$ esu $\left(-1.3 \times 10^{-40} \mathrm{C} \mathrm{m}^{2}\right)$. [20] The 9-6 potential parameters were adjusted so that the experimental values of the second virial coefficient, $B(T)$, were realized between $110 \mathrm{~K}$ and $400 \mathrm{~K}$ [21] within the standard error of the Monte Carlo procedure used to evaluate $B(T)$. The sixth power combining rules, eqs. 6 and 7 , were used to obtain the values for the unlike sites listed in Table 1 . Since both the pyridine and oxygen potentials are adapted to the conditions of interest, the solubility calculation is primarily a test of the combining rules if the essential physics for solute-solvent interactions is contained in the model potentials for the pure components.

The oxygen-pyridine interactions that enter the coupling expression are assumed to have a modified Lennard-Jones plus charge-quadrupole interaction. As noted above, the inverse power terms are modified by $r$ being replaced by $r_{a}=\alpha(1-\lambda)^{2}+r$ so that singularities are avoided. [6] The cutoff length $\alpha$ was arbitrarily set to $0.158 \mathrm{~nm}$, a value that is on the order of $1 / 2$ of the position of the zeros of the Lennard-Jones potentials. The sensitivity of the results to the value of $\alpha$ is discussed in the appendix. The result is

$$
U(\lambda, r)=\lambda^{2} \sum_{\text {sites }}\left[\epsilon\left[\left(r_{0} / r_{a}\right)^{12}-2\left(r_{0} / r_{a}\right)^{6}\right]+q Q r^{2} / r_{a}^{5}\left[3(\hat{z} \cdot \mathbf{r})^{2}-r^{2}\right]\right]
$$

where $\hat{z}$ is a unit vector lying along the axis of the oxygen molecule, $\mathrm{q}$ is the charge on the pyridine site and $\mathrm{Q}$ is the quadrupole moment of the oxygen molecule. The total interaction, $U$, is a sum over all pyridine molecule sites and over the two oxygen sites with 12-6 interactions and the center of mass oxygen site with the charge-quadupole interaction. [22] The 12-6 form was arbitrarily chosen after some initial results indicated possible problems with the softer repulsion of the 9-6 form. This form for the 12-6 potential has $r_{0}$ the position of the minimum of the potential and $\epsilon$ as the well depth, just as in the 9-6 case. As will be shown in the next section, the problem was not with the 9-6 form but with the combining rules. The zero of the Lennard-Jones potential, $\sigma$ satisfies $r_{0}=2^{1 / 6} \sigma$.

Molecular dynamics is used to sample phase space in this work. Since only thermodynamic quantities are of interest, NPT Monte Carlo simulation could also be used. [23] Which sampling method is used is largely a matter of individual choice.

\section{Results}

The first set of results are for the parameters in Table 1. As shown below, the prediction of the solubility based on those parameters is significantly smaller than the experimental 
value.

The evaluations of $\langle\partial U(\lambda) / \partial \lambda\rangle_{\lambda}$ have been performed for 0.1 increments of $\lambda$ from 0.0 to 1.0 plus a few additional values of $\lambda$ to better define how the $\langle\partial U / \partial \lambda\rangle$ goes to zero as $\lambda$ goes to zero and to fully resolve the maximum that occurs in the vicinity of $\lambda=1 / 2$. This proved sufficient to obtain reliable estimates of the integral. The uncertainties in $\langle\partial U(\lambda) / \partial \lambda\rangle_{\lambda}$ are obtained using block averaging [24] as described in detail in the appendix.

The integrand of eq. 2 for the parameters listed in Table 1 is shown in Fig. 2. The integral has the value 6.76. When the experimental values for the number density of oxygen gas and liquid pyridine $\left(n_{v}=2.245 \times 10^{19} \mathrm{~cm}^{-3}[25], n_{l}=7.39 \times 10^{21} \mathrm{~cm}^{-3}\right.$ [18] $)$ are used in eq. 3 , the solubility $s=3.8 \times 10^{-6}$. This is much smaller than the experimental value of $4.5 \times 10^{-4}[9]$

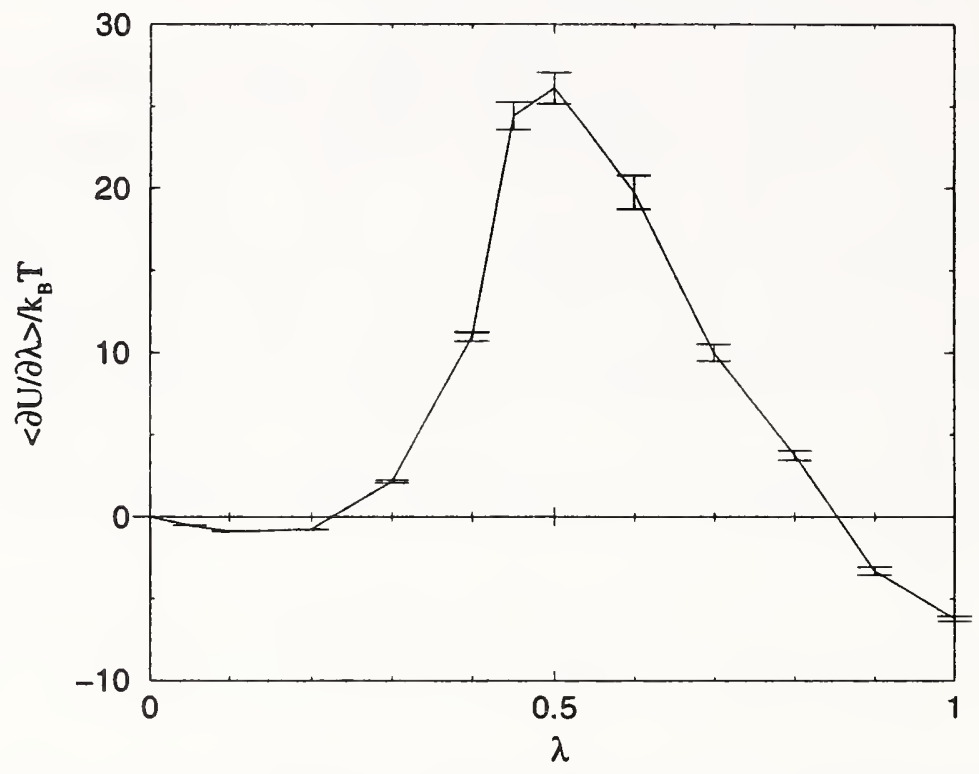

Fig. 2. The integrand of eq. 2 obtained using the parameters listed in Table 1 exhibits a maximum around $\lambda=1 / 2$.

The discrepancy between the calculated value of the solublity and the experimental value is due to the excessively large value of the coupling integral. It is apparent from Fig. 2 that in order to obtain agreement with experiment the large positive peak around $\lambda=1 / 2$ should be reduced and/or the negative region for larger values of $\lambda$ be increased. Since we are using purely empirical potentials for the 12-6 interactions, it is appropriate to experiment with other values of the potential parameters. The values for the charges on the pyridine molecule sites and the quadrupole moment of the oxygen molecule are based on quantum chemistry calculations and experimental measurements so modifying them is not advisable at this time. Also, the 9-6 parameters for pyridine-pyridine and oxygen-oxygen interactions are dependent on the values for the charges and the quadrupole moment. Therefore, the 
12-6 parameters are the simplest ones to adjust. Some initial tests indicated that modifying the size parameters, $r_{0}(a b)$, would have large influence on the results. Instead, the more fruitful approach, one that does not lead to such large changes, is to vary the well depth parameters, $\epsilon(a b)$.

As an initial exercise in parameter adjustment, the $\epsilon(\mathrm{OC}), \epsilon(\mathrm{OH})$, and $\epsilon(\mathrm{ON})$ parameters were arbitrarily increased to $47.2 \mathrm{~K}, 30.4 \mathrm{~K}$, and $80.2 \mathrm{~K}$ respectively, about twice the values in Table 1. The resulting integrand is shown in Fig. 3. The value of the integral is 1.16 and the solubility is $1 \times 10^{-3}$. Therefore the well depth parameters were increased by too much.

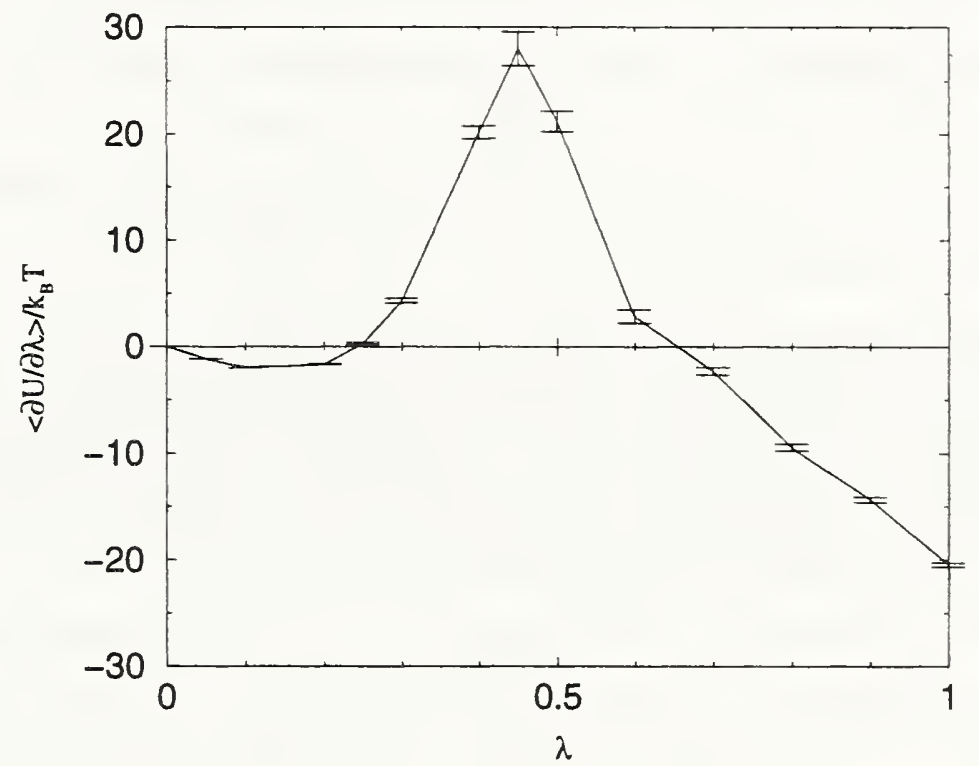

Fig. 3. The integrand of eq. 2 obtained using well depth parameters that are about twice the values in Table 1.

Next, a series of calculations were performed that indicated that the integrand was not sensitive to the value of $\epsilon(\mathrm{OH})$ and was most sensitive to the value of the $\epsilon(\mathrm{OC})$. This then lead to further calculations that narrowed the values to $54.5 \mathrm{~K}, 14.0 \mathrm{~K}$, and $60.2 \mathrm{~K}$ for $\epsilon(\mathrm{OC}), \epsilon(\mathrm{OH})$, and $\epsilon(\mathrm{ON})$ respectively. The resulting integrand is shown in Fig. 4. The value of the integral is 2.0 and the solubility is $4.5 \times 10^{-4}$. This is essentially the same as the experimental value. One should keep in mind that the uncertainty in the estimate for the integral is on the order of $10 \%$ with a corresponding uncertainty of about $10 \%$ in the solubility. There is no point to further adjustment of the well depth parameters at this time. These well depth parameters are not accessible by the usual combining rules.

\section{Discussion}

The Kirkwood charging parameter approach to estimating the chemical potential of an infinitely dilute solute has been implemented. The emphasis here will be on describing the 


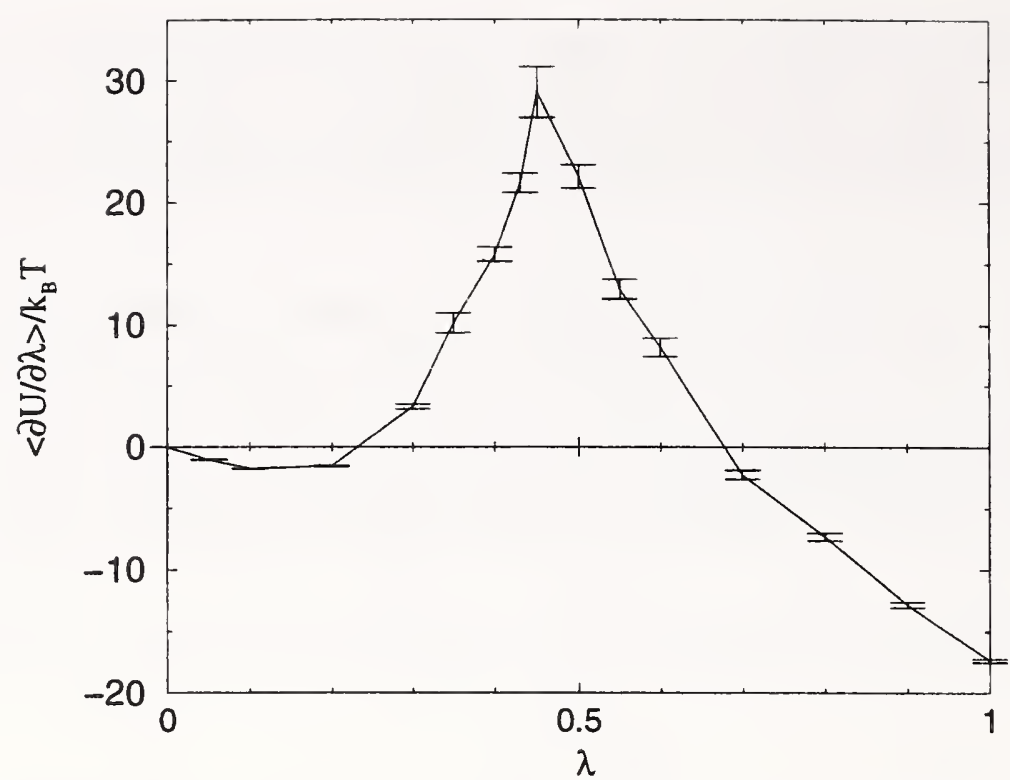

Fig. 4. The integrand of eq. 2 for the $\epsilon$ set that leads to agreement with the experimental value of the solubility.

details of the implementation and not so much on the explicit results. The results indicate that the use of combining rules to obtain solvent-solute potential parameters when the two species are not similar is not likely to yield satisfactory results. Instead, one needs to attempt to determine those quantities directly as discussed below.

The charging approach requires that the integrand, $\langle\partial U(\lambda) / \partial \lambda\rangle_{\lambda}$, be obtained for enough values of the charging parameter $\lambda$ so that the integral in eq. 2 can be obtained with good accuracy. For the case at hand shown in Fig. 4, this means that the resolution around the maximum must be on the order 0.05 while the other parts of the integrand can be evaluated with 0.10 resolution. It is also important that the charging potentials contain a cutoff length $\alpha$ that shields the system from the singularities in the potentials. The value for $\alpha$ should be chosen so that the maximum is not so large as to make it necessary to use very fine resolution. As discussed in the appendix, the value of $\alpha=0.158 \mathrm{~nm}$, about $1 / 2$ the position of the zern of the Lennard-Jones potentials is a reasonably good choice for the pyridine-oxygen system.

The steps that are needed to implement this approach to the determination of the solubility of a volatile compound in a solvent are worth examining in a bit more detail. First, one needs a solvent potential that will produce satisfactory PVT properties for the liquid over the region of interest. Alternatively, one could adopt empirical potentials that produce reliable vapor pressures. This might change the energy parameters, $\epsilon(\mathrm{ab})$, so that the combining rules would be useful. This possibility has not been examined.

The next step, one that is difficult to implement on the basis of fitting to physical properties such as the equation of state, is to determine the solute-solvent potential. In the current 
work, this was done empirically by a trial and error approach using the known value of the solubility as the target. Since the solubility is the quantity of interest, this amounts to curve fitting with unknown ability to predict the solubility for other conditions. An alternative would be to perform quantum chemistry calculations of the interaction energy of a solute-solvent pair for a large number of configurations and then parameterize the resulting energy surface in the form of a set of effective pair potentials. A quantum chemistry approach could guide the development of a more detailed description of the electrostatic interactions. In particular, the possiblity of induced polarization could be considered. Another possibilty that has recently been proposed would be to use chemical shift measurements, if available, to estimate the solute-solvent interactions. [26]

Finally, it is essential that the effective solute-solvent pair potentials be modified, as done in eq. 8 so that any small separation singularities are screened for small values of the charging parameter $\lambda$.

Once the potentials are in hand, then a series of simulations need to be performed to determine the $\lambda$ variation of $\langle\partial U(\lambda) / \partial \lambda\rangle_{\lambda}$. The initial set would sketch out the $\lambda$ variation with a resolution of 0.1 . Once these 10 simulations are completed, any gaps in the resolution can be filled in with additional simulations. While this is a tedious undertaking, it is certain to provide a reliable estimate of the solubility for the model potentials used. The test for convergence of a given run should be the convergence of the running average as illustrated in Fig. A1 in the appendix.

In summary, the steps to be followed when using the Kirkwood charging approach to determining the solubility of a gas in a liquid have been examined. The importance of an independent determination of the solute-solvent interaction can not be overemphasized. Reliance on combining rules can result in estimates of the solubility that are in serious error.

Although it is outside the main topic of this report, it is interesting to compare the solubility of $\mathrm{N}_{2}$ in pyridine with that of $\mathrm{O}_{2}$. At $298 \mathrm{~K}$, the solubility of $\mathrm{N}_{2}$ in pyridine is $2.5 \times 10^{-4}$, [27] slightly more than $1 / 2$ that of $\mathrm{O}_{2}$. We also note that the quadrupole moment of $\mathrm{N}_{2}$ is 3.5 times larger than that of oxygen. [20] The significance of this difference, if any, in determining the solubility of the gases is not known.

\section{Appendix}

\section{Non-ideal gas formulation}

If the solute in the vapor phase is not an ideal gas, then eq. 2 must be modified to include the nonideal contribution to the chemical potential. In general, corrections to ideal gas behavior become important at higher densities. The presure where this occurs depends on the specific gas and on the temperature. If the pressure can be represented by a virial 
series, $[28]$

$$
p=k_{B} T\left[n_{v}+B_{2}(T) n_{v}^{2}+B_{3}(T) n_{v}^{3}+\ldots\right]
$$

then it follows that the chemical potential can be represented by

$$
\mu=k_{B} T\left[\ln \left(n_{v} \Lambda^{3}\right)+\sum_{k \geq 1}\left(\frac{k+1}{k}\right) B_{k+1}(T) n_{v}^{k}\right]
$$

Now equating the chemical potentials given by eqs. 1 and $\mathrm{A} 1$ yields the modified form of eq. 2 ,

$$
\ln \left(n_{s} / n_{v}\right)=-\frac{1}{k_{B} T} \int_{0}^{1}\langle\partial U(\lambda) / \partial \lambda\rangle_{\lambda} d \lambda+\sum_{k \geq 1}\left(\frac{k+1}{k}\right) B_{k+1}(T) n_{v}^{k}
$$

This equation would be used when the pressure of oxygen is greater than $0.1 \mathrm{MPa}$.

\section{Cutoff length}

As noted in section 2, the cutoff length, $\alpha$ was arbitrarily assigned a value of $0.158 \mathrm{~nm}$ which is about $1 / 2$ the value of the zero of the Lennard-Jones potentials. Two further tests were made for the values of the $\epsilon$ 's that yielded agreement with the experimental value of the solubility. In these tests, $\alpha$ was given the values $0.079 \mathrm{~nm}$ and $0.237 \mathrm{~nm}$. The resulting integrands are shown in the Fig. A1.

No attempt was made to fully resolve the two test cases, instead the intent is show how the derivatives vary with the length $\alpha$. Note that the peak of the case with $\alpha=0.237 \mathrm{~nm}$ has not been fully resolved. When $\alpha$ is small, the curve should be more fully resolved than was done here since the area under that curve, as shown, is significantly larger than the area under the other two curves.

From these examples, it appears that putting $\alpha$ in the range between $1 / 2$ and $3 / 4$ of the position of the zero of the interaction potentials is a satisfactory choice. Smaller values of $\alpha$ do not appear to provide adequate screening the singularities as indicated by the relatively large positive region for $\lambda \leq 0.6$ in Fig. A1.

Sampling and convergence issues

The estimates for $\langle\partial U(\lambda) / \partial \lambda\rangle_{\lambda}$ were made for well stabilized systems for each value of the charging parameter $\lambda$. In practice this meant that once a value of $\lambda$ had been assigned, the system was evolved for at least 10 ps before a production run of 100 ps was made. During 


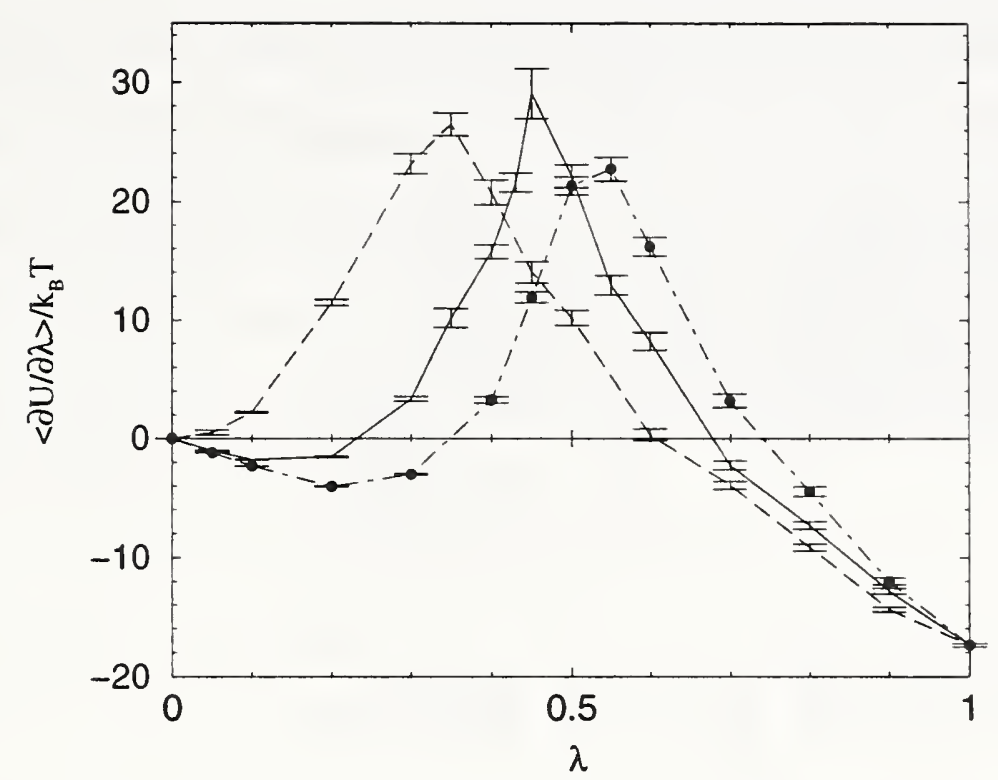

Fig. A1. The expectations of $\langle\partial U(\lambda) / \partial \lambda\rangle_{\lambda} / k_{B} T$ for three values of the cutoff length, $\alpha$ are shown here. The solid line is for $\alpha=0.158 \mathrm{~nm}$ (also shown in Fig. 4), the dashed line is for $\alpha=0.079 \mathrm{~nm}$, and the long-short dashed line with solid symbols is for $\alpha=0.237 \mathrm{~nm}$.

the run, block averages of 1 ps duration were made of the derivative. [24] A typical case is shown in the Fig. A2 for the sequence of time averages over a 100 ps run. Also shown in the figure is the running average, that is the average at time $t$ of the block averages up to that time. The convergence of this running average to the overall time average is an indication that adequate sampling has been performed. The type $A$ standard error for independent observations is used to estimate the uncertainty in the average value of the derivative. [29] This illustrates the need for runs of 100 ps duration as the convergence in this example is at best marginal at $100 \mathrm{ps}$. The convergence of the running average to the overall time average, indicated by a nearly horizontal segment of the curve for longer times, should be checked for each value of $\lambda$ generated. 


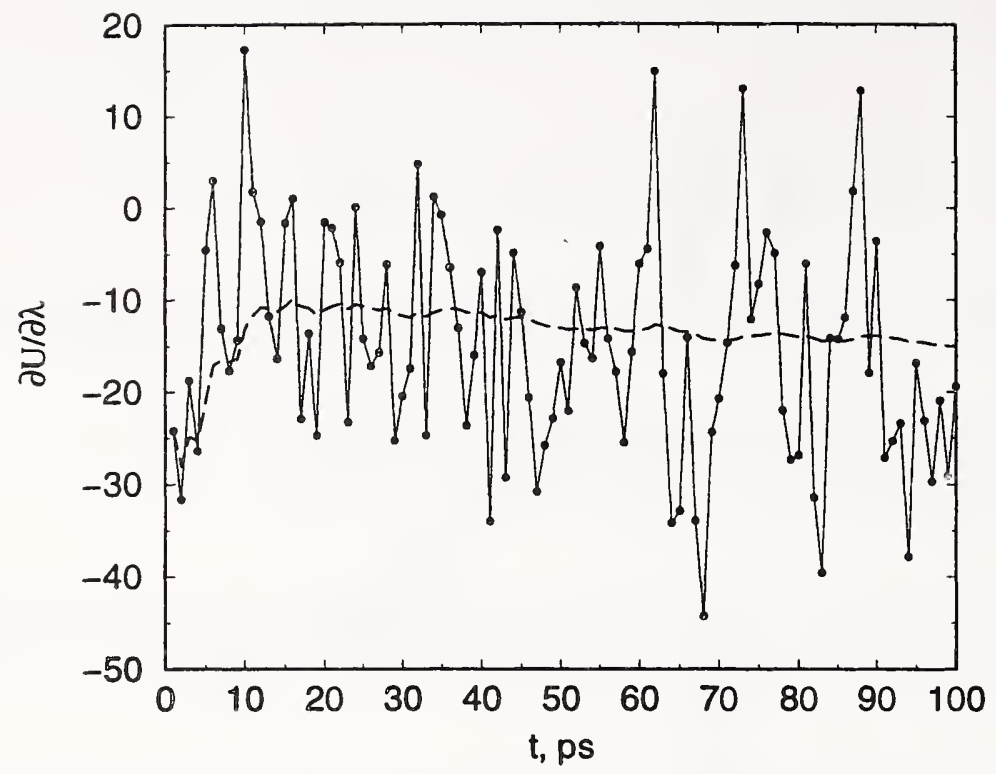

Fig. A2. The time series for $\langle\partial U(\lambda) / \partial \lambda\rangle_{\lambda}$ (solid line and filled circles $\bullet$ ) and the cumulative average (dashed line) for the case with $\alpha=0.79 \mathrm{~nm}$ and $\lambda=0.7$. The overall average in the derivative for this case is $-15.0 \pm 1.2$. This is a marginally converged case as the running average curve is slightly tilted downward.

\section{References}

[1] Hill, T. L., Statistical Mechanics (McGraw Hill, New York, 1956), p. 192.

[2] Cástellan, G. W., Physical Chemistry: $2^{\text {nd }}$ edition (Addison Wesley, Reading, MA, 1971), pp. 321-322.

[3] Wilson, A. H., Thermodynamics and Statistical Mechanics (University Press, Cambridge, 1960), p. 230.

[4] Swope, W. C. and Andersen, H. C., J. Phys. Chem. 88, 6548 (1984).

[5] Ferrario, M., Ciccotti, G., Spohr, E., Cartailler, T., and Turq, P., J. Chem. Phys. 117, 4947 (2002).

[6] Beutler, T. C., Mark, A. E., van Schaik, R. C., Gerber, P. R., and van Gunsteren, W. F., Chem. Phys. Letters 222, 529 (1994).

[7] Widom, B., J. Chem. Phys. 39, 2802 (1963).

[8] Frenkel, D. and Smit, B., Understanding Molecular Simulation (Academic Press, New York, 2002), pp. 176-177, 2nd edition.

[9] Battino, R., ed., Solubility Data Series, Oxygen and Ozone (Pergamon Press, New York, 1981), volume 7, p. 352.

[10] Martin, M. G. and Siepmann, J. I., Theor. Chem. Acc. 99, 347 (1998). 
[11] Martyna, G. J., Tobias, D. J., and Klein, M. L., J. Chem. Phys. 101, 4177 (1994).

[12] Martys, N. S. and Mountain, R. D., Phys. Rev. E 59, 3733 (1999).

[13] Singer, K., Taylor, A., and Singer, J. V. L., Mol. Phys. 33, 1757 (1977).

[14] Bak, B., Hasen-Nygaard, L., and Rastrup-Andersen, J., J. Mol. Spect. 2, 316 (1958).

[15] Johnson III, R. D., Computational Chemistry Comparison and Benchmark Data Base, http://srdata.nist.gov/cccbdb.

[16] Sun, H., Fluid Phase Equilibria yy, a (2003), in press.

[17] Waldman, M. and Hagler, A. T., J. Comp. Chem. 14, 1077 (1993).

[18] Fury, M., Munie, G., and Jonas, J., J. Chem. Phys. 70, 1260 (1979).

[19] Perng, B.-C., Sasaki, S., Ladanyi, B. M., Everitt, K. F., and Skinner, J. L., Chem. Phys. Letters 348, 491 (2001).

[20] Gray, C. G. and Gubbins, K. E., Theory of Molecular Fluids, Vol. 1: Fundamentals (Clarendon Press, Oxford, 1984), p. 578.

[21] Dymond, J. H. and Smith, E. B., The Virial Coefficients of Gases: A Critical Complilation (Clarendon Press, Oxford, 1969), pp. 200-202.

[22] Price, S. L., Stone, A. J., and Alderton, M., Mol. Phys. 52, 987 (1984).

[23] Allen, M. P. and Tildesley, D. J., Computer Simulation of Liquids (Oxford University Press, Oxford, UK, 1987), pp. 123-126.

[24] Bishop, M. and Frinks, S., J. Chem. Phys. 87, 3675 (1987).

[25] Weber, L. A., J. Res. NBS 74A, 93 (1970).

[26] Jameson, C. J. and Murad, S., Chem. Phys. Letters 380, 556 (2003).

[27] Battino, R., ed., Solubility Data Series, Nitrogen and Air (Pergamon Press, New York, 1982), volume 10, p. 263.

[28] Hill, T. L., An Introduction to Statistical Thermodynamics (Addison-Wesley, Reading, MA, 1960), pp. 264-266.

[29] Taylor, B. N. and Kuyatt, C. E., Guidelines for Evaluating and Expressing the Uncertainty of NIST Measurement Results, NIST Technical Note 1297, 1994 Edition, National Institute of Standards and Technology (1994). 


